\title{
Interphotoreceptor retinoid-binding protein (IRBP) is downregulated at early stages of diabetic retinopathy
}

\author{
M. Garcia-Ramírez • C. Hernández • M. Villarroel • \\ F. Canals • M. A. Alonso $\cdot$ R. Fortuny $\cdot$ L. Masmiquel • \\ A. Navarro • J. García-Arumí • R. Simó
}

Received: 27 July 2009 /Accepted: 2 September 2009 /Published online: 13 October 2009

(C) Springer-Verlag 2009

\begin{abstract}
Aims/hypothesis Interphotoreceptor retinoid-binding protein (IRBP) plays a major role in the visual cycle and is essential to the maintenance of photoreceptors. The aim of this study was to determine whether a decrease in IRBP production exists in the early stages of diabetic retinopathy. Methods Vitreous samples from diabetic patients with proliferative and non-proliferative diabetic retinopathy
\end{abstract}

M. Garcia-Ramírez $\cdot$ C. Hernández $\cdot$ M. Villarroel $\cdot$ R. Simó $(\square)$ CIBER for Diabetes and Associated Metabolic Diseases (CIBERDEM), Diabetes and Metabolism Research Unit, Institut de Recerca Hospital Universitari Vall d'Hebron, Universitat Autònoma de Barcelona Pg. Vall d'Hebron 119-129, 08035 Barcelona, Spain

e-mail: rsimo@ir.vhebron.net

F. Canals

Proteomics Laboratory, Medical Oncology Research Program, Institut de Recerca, Hospital Universitari Vall d'Hebron,

Barcelona, Spain

M. A. Alonso

Department of Ophthalmology, Hospital de Son Llàtzer,

Palma, Spain

R. Fortuny

Department of Biochemistry, Hospital de Son Llàtzer,

Palma, Spain

L. Masmiquel

Endocrinology Service, Hospital de Son Llàtzer,

Palma, Spain

\author{
A. Navarro \\ Blood and Tissue Bank, Hospital Universitari Vall d'Hebron, \\ Barcelona, Spain \\ J. García-Arumí \\ Service of Ophthalmology, Hospital Universitari Vall d'Hebron, \\ Barcelona, Spain
}

(PDR, NPDR), and from non-diabetic patients with macular hole (control group) were selected for IRBP quantitative assessment by proteomic analysis (fluorescence-based difference gel electrophoresis) and western blot. Human post mortem eyes $(n=16)$ from diabetic donors without clinically detectable retinopathy and from non-diabetic donors $(n=16)$ were used to determine IRBP (also known as RBP3) mRNA levels (RT-PCR) and protein content (western blot and confocal microscopy). Retinal neurodegeneration was assessed by measuring glial fibrillar acidic protein (GFAP) and the apoptotic rate. Y79 human retinoblastoma cells were used to test the effects of glucose, TNF- $\alpha$ and IL-1 $\beta$ on IRBP expression and IRBP levels. Results Intravitreous IRBP concentration was significantly lower in $\mathrm{PDR}<\mathrm{NPDR}<$ control in proteomic and western blot analysis. IRBP mRNA levels and IRBP protein content were significantly lower in the retinas from diabetic donors than in those from non-diabetic donors. Increased GFAP and a higher degree of apoptosis were observed in diabetic retinas compared with non-diabetic retinas. A dosedependent downregulation of IRBP mRNA expression and IRBP content was detected with glucose, TNF- $\alpha$ and IL-1 $\beta$ in cultures of Y79 human retinoblastoma cells.

Conclusions/interpretation Underproduction of IRBP is an early event in the human diabetic retina and is associated with retinal neurodegeneration. The mechanisms leading to this deficit deserve further investigation.

Keywords Diabetic retinopathy · Human study . Interphotoreceptor retinoid-binding protein .

Neurodegeneration $\cdot$ Retina $\cdot$ Vitreous fluid

\section{Abbreviations}

DIGE Fluorescence-based difference gel electrophoresis GFAP Glial fibrillar acidic protein 
IRBP Interphotoreceptor retinoid-binding protein

NPDR Non-proliferative diabetic retinopathy

PDR Proliferative diabetic retinopathy

RPE Retinal pigment epithelium

TdT Terminal deoxynucleotidyl transferase

\section{Introduction}

Diabetic retinopathy has been classically considered to be a microcirculatory disease of the retina. However, before any microcirculatory abnormalities can be detected, retinal neurodegeneration is already present [1-4]. In fact, we have detected apoptosis and glial activation, two characteristic features of retinal neurodegeneration, in retinas from diabetic donors, which were free of microcirculatory abnormalities in ophthalmoscopic examinations performed in the 2 years preceding death $[5,6]$. Therefore, the study of the mechanisms that lead to neurodegeneration and of their effects on essential proteins involved in the visual cycle could be crucial for identifying new therapeutic targets in the early stages of diabetic retinopathy.

In the vertebrate retina, vision is initiated and maintained by the photolysis and regeneration, respectively, of lightsensitive pigments in the disk membranes of the photoreceptor outer segments. This cyclical process depends on an exchange of retinoids between the photoreceptors and the retinal pigment epithelium (RPE). There is considerable evidence that the transport of retinoids between these cellular compartments is mediated by the interphotoreceptor retinoid-binding protein (IRBP), a large glycoprotein synthesised in the photoreceptors and extruded into the interphotoreceptor matrix that fills the subretinal space $[7,8]$. Apart from participating in the visual cycle, IRBP is important in fatty acid transport and is essential to the maintenance of photoreceptors $[9,10]$. In this regard, a reduction of IRBP may precede the loss of photoreceptors seen in some animal models of hereditary retinal degeneration $[11,12]$. In addition, knockout $\left(\operatorname{Irbp}^{-/}\right)$mice revealed a loss of photoreceptors and profound changes in the structural integrity of the receptor outer segments $[10,13]$. Recently, a homozygous missense mutation in the IRBP gene (also known as $R B P 3$ ) has been associated with autosomal recessive retinitis pigmentosa in children [14].

We recently compared the protein profiles of human vitreous from diabetic patients with proliferative diabetic retinopathy (PDR) with the vitreous fluid from non-diabetic patients with macular holes using the fluorescence-based difference gel electrophoresis (DIGE) strategy [15]. Our results provided evidence that IRBP is among the lowest produced proteins in the vitreous fluid of patients with PDR. However, it is unknown whether this finding is also present in the early stages of diabetic retinopathy. This is important because a deficit of IRBP could be involved in the impairment of vision that occurs in diabetic patients even before clinical diabetic retinopathy can be detected. In addition, the lower IRBP content could lead to photoreceptor damage, which is one of the components of retinal neurodegeneration.

On this basis, the aim of the present study was to determine whether decreased IRBP production also exists in the early stages of diabetic retinopathy. For this purpose we used vitreous fluid from diabetic patients with nonproliferative diabetic retinopathy (NPDR) and retinas from diabetic donors without clinically detectable diabetic retinopathy.

\section{Methods}

Participants and sample selection

Vitreous fluid Samples of vitreous from four type 2 diabetic patients with PDR, four type 2 diabetic patients with NPDR and from eight non-diabetic patients with idiopathic macular hole (control group), all closely matched by age, were selected from our vitreous bank for proteomic analysis.

An additional 30 samples (ten PDR, ten NPDR and ten macular hole, matched by age) were used for western blot analysis. Exclusion criteria were: (1) previous vitreoretinal surgery; (2) photocoagulation in the preceding 6 months; (3) recent vitreous haemorrhage (less than 3 months before vitrectomy) or intravitreous haemoglobin higher than $5 \mathrm{mg} / \mathrm{ml}$; (4) renal failure (creatinine $\geq 120 \mu \mathrm{mol} / \mathrm{l}$ ); and (5) other chronic diseases apart from diabetes. For diabetic retinopathy classification we used the International Clinical Diabetic Retinopathy Severity Scale [16]. In accordance with this classification, NPDR samples were from patients with mild $(n=8)$ and moderate $(n=2)$ NPDR. Diabetic nephropathy was defined according to the criteria of the American Diabetes Association [17]. The main characteristics of controls, NPDR and PDR patients are summarised in Table 1.

Details of vitrectomy and sample collection have been previously described [18].

\section{Retinas}

We obtained 16 human post mortem eyes from eight diabetic donors (age 66.4 \pm 5.9 years). Eyes were free of fundoscopic abnormalities in ophthalmological examinations performed during the preceding 2 years. Eye cups $(n=$ 16) obtained from eight non-diabetic donors matched by age (age 67.6 \pm 6.9 years) were used as the control group. 
Table 1 Main clinical features of participants included in the study

\begin{tabular}{|c|c|c|c|c|}
\hline \multirow[t]{2}{*}{ Variables } & \multicolumn{3}{|c|}{ Participant groups } & \multirow[t]{2}{*}{$p$ value } \\
\hline & $\begin{array}{l}\text { With PDR } \\
10\end{array}$ & $\begin{array}{l}\text { With NPDR } \\
10\end{array}$ & $\begin{array}{l}\text { Control group } \\
10\end{array}$ & \\
\hline Age (years) & $60.3 \pm 15.3$ & $61.3 \pm 16.2$ & $59.6 \pm 10.7$ & $0.85^{\mathrm{a}}$ \\
\hline Sex (men/women) & $5 / 5$ & $5 / 5$ & $4 / 6$ & $0.65^{\mathrm{a}}$ \\
\hline Hypertension (\%) & 70 & 60 & 50 & $0.36^{\mathrm{a}}$ \\
\hline Duration of diabetes (years) & $15.9 \pm 6.2$ & $6.9 \pm 3.1$ & - & $<0.001^{\mathrm{b}}$ \\
\hline $\mathrm{HbA}_{1 \mathrm{c}}(\%)^{\mathrm{c}}$ & $8.1 \pm 2.9$ & $7.9 \pm 3.5$ & - & $0.47^{\mathrm{b}}$ \\
\hline Nephropathy $(\%)^{\mathrm{d}}$ & 60 & 20 & - & $0.08^{\mathrm{b}}$ \\
\hline Neuropathy (\%) & 40 & 10 & - & $0.15^{\mathrm{b}}$ \\
\hline Macroangiopathy (\%) & 50 & 30 & - & $0.32^{\mathrm{b}}$ \\
\hline
\end{tabular}

Unless otherwise indicated, data are expressed as the mean \pm SD

${ }^{a}$ For comparison among the three groups

${ }^{\mathrm{b}}$ For comparison between PDR and NPDR participants

${ }^{\mathrm{c}}$ Mean of the values obtained in the preceding year

${ }^{\mathrm{d}}$ Diabetic nephropathy was defined according to the criteria of the American Diabetes Association [17], but patients with renal failure (creatinine $\geq 120 \mu \mathrm{mol} / \mathrm{l})$ were excluded

The main clinical features and causes of death of diabetic and non-diabetic donors are summarised in Table 2.

The time elapsing from death to eye enucleation was less than $4 \mathrm{~h}$. After enucleation, one eye of each donor was snap-frozen at $-80^{\circ} \mathrm{C}$ and stored until assayed for mRNA and western blot analyses. The other was fixed in $4 \%$ (wt/vol.) paraformaldehyde and embedded in paraffin for the immunohistochemical study.

All ocular tissues were obtained and processed following the recommendations of the Declaration of Helsinki for research involving human tissue and those of the Directive of the European Parliament and the Council of the European Union (Directive 2004/23/EC). In addition this study was approved by the Ethics Committee of our hospital.

Sample preparation, DIGE proteomic analysis and protein identification Sample preparation along with details of DIGE proteomic analysis and protein identification by mass spectrometry have been described elsewhere [15].

\section{IRBP assessment}

RNA extraction and IRBP $m R N A$ quantification Neuroretinas and RPE were removed under microscopic dissection of isolated eye cups from donors. Total RNA was obtained from isolated human neuroretinas of eye donors using a kit (RNeasy Mini; Quiagen Distributor IZASA, Barcelona, Spain) according to the manufacturer's protocol.
We used $1 \mu \mathrm{g}$ total RNA for reverse transcription. Quantitative real-time PCR was performed with $40 \mathrm{ng}$ of cDNA and TaqMan Universal Mastermix (Applied Biosystems, Madrid, Spain) in an Applied Biosystems device (7900 model). TaqMan pre-made gene expression assays (Applied Biosystems) were used for specific gene amplifi-

Table 2 Clinical features of diabetic and non-diabetic donors included in the study

\begin{tabular}{llll}
\hline Variables & $\begin{array}{l}\text { Diabetic } \\
\text { donors }^{\mathrm{a}} \\
n\end{array}$ & $\begin{array}{l}\text { Non-diabetic } \\
\text { donors } \\
8\end{array}$ & $p$ value \\
\hline Age (years) & $66.4 \pm 5.9$ & $67.6 \pm 6.9$ & 0.47 \\
Sex (men/women) & $5 / 3$ & $6 / 2$ & 0.50 \\
Diabetes duration (years) & $5.8 \pm 3.1$ & - & \\
HbA $_{1 \mathrm{c}}(\%)^{\mathrm{b}}$ & $7.4 \pm 0.8$ & - & \\
Diabetes treatment & & & \\
$\quad$ Diet only & 3 & & \\
Oral agents & 5 & & \\
Insulin & 0 & 3 & \\
Cause of death & & 4 & \\
Cardiovascular disease & 5 & 1 & \\
Malignant neoplasm & 3 & 0 & \\
$\quad$ Traffic accident & 0 & & \\
\hline
\end{tabular}

Data are expressed as $n$ or as the mean $\pm \mathrm{SD}$

${ }^{\text {a }}$ All diabetic donors had type 2 diabetes

${ }^{\mathrm{b}}$ Mean of the values obtained in the preceding 2 years 
cation (human IRBP: Hs00161253 m1). Each sample was assayed in duplicate and negative controls were included in each experiment. Automatic relative quantification was obtained with a software package (ABI Prism 7900 SDS; Applied Biosystems) using $\beta$-actin as the endogenous gene expression reference (Hs9999903_m1; Applied Biosystems).

Western blot analysis IRBP was assessed in the vitreous fluid of diabetic and control patients, and in the neuroretina from donors. Total protein extracts from the neuroretina were quantified using an assay (Bradford Protein Assay; Bio-Rad, Barcelona, Spain). Equal amounts of protein $(5 \mu \mathrm{g})$ were loaded on a $7.5 \%$ (vol./vol.) SDSpolyacrylamide gel and electroblotted on to nitrocellulose membranes (Immobilon; Millipore, Barcelona, Spain). Membranes were probed with IRBP antibodies (kindly provided by J. M. Nickerson, Emory University, Atlanta, GA, USA) at a dilution of 1:2,000. Anti human $\beta$-actin (Calbiochem, Bionova, Madrid, Spain) was used for normalisation. Blots were developed by chemoluminescence (Supersignal West Dura Extended Duration Substrate; Pierce, Madrid, Spain). Densitometry of the developed bands was obtained in a calibrated GS- 800 device (Bio-Rad).

Immunohistochemistry for IRBP Neuroretina sections $(6 \mu \mathrm{m})$ were dewaxed following standardised methods. Sections were permeabilised and blocked for $1 \mathrm{~h}$ in PBS buffer with $0.05 \%$ (vol./vol.) Tween and $2 \%$ (wt/vol.) BSA. Primary antibodies (anti-IRBP and anti-glial fibrillar acidic protein [GFAP] [Sigma, Madrid, Spain]) were incubated overnight at $4^{\circ} \mathrm{C}$ in the same blocking buffer. Afterwards, sections were extensively washed, then incubated with Alexa Fluor 488 or Alexa Fluor 568 anti-rabbit (1:200; Molecular Probes, Invitrogen, Madrid, Spain) for $1 \mathrm{~h}$. A negative control section was performed by omitting the corresponding antiserum from the primary antibody solution. Double staining was performed including two antibodies in the same section. After three washes in PBS buffer, sections were mounted on fluorescence mounting medium with DAPI (H-1200; Vector, CA, USA) and observed under a spectral confocal microscope (FV1000; Olympus, Madrid, Spain). Quantification of immunofluorescence was performed directly on confocal images of 1.024 pixels with an Olympus Fluoview imaging system (Olympus, Barcelona, Spain). To quantify IRBP in the neuroretina, the total fluorescence intensity values corresponding to ten field frame images $(40 \times$ numerical aperture 0.9 ) of each retina sample were measured. These results were then normalised taking into account the area analysed. All these calculations were made using specific software (Fluoview ASW 1.4; Olympus).
Measurements of retinal neurodegeneration

Apoptosis: TUNEL Neuroretina sections were processed using an in situ apoptosis detection kit (ApoTaq Plus Fluorescein; Millipore, Madrid, Spain) according to the manufacturer's protocol. Briefly, sections were deparaffinised with xylene and ethanol, and the tissue pretreated with proteinase $\mathrm{K}(20 \mu \mathrm{g} / \mathrm{ml})$ for $15 \mathrm{~min}$ before digoxigenin-labelled dUTP was applied in the presence of the terminal deoxynucleotidyl transferase (TdT) enzyme (TdT concentration was changed to half the recommended dilution). Nuclear staining in apoptotic cells was detected by FITC staining with anti-digoxigenin conjugate. To counterstain, propidium iodide antifade solution (Millipore) was used as a mounting media. A non-specific control was included by omitting the TdT enzyme in the incubation step. Positive controls were incubated with DNAse I prior to incubation with TdT. Quantification was performed by counting individually fluorescence-labelled cells in three sections per sample. The apoptotic index was calculated by dividing the total number of apoptotic cells by the total number of cells in each layer and in a $0.5 \mathrm{~cm}^{2}$ area. To avoid false positive results, only TUNEL-positive cells in which nuclear fragmentation and/or condensation was present were rated as apoptotic. For this purpose we used inter-differential contrast (phase-contrast microscopy) and propidium-iodide immunofluorescence.

Glial activation: GFAP immunofluorescence Tissue sections were incubated overnight at $4^{\circ} \mathrm{C}$ with the primary antibody anti-human GFAP (1:200; Sigma). After washing, sections were incubated with Alexa Fluor 488 (Molecular Probes, Eugene, OR, USA) secondary antibody for $1 \mathrm{~h}$. GFAP immunofluorescence in the neuroretina was quantified using a laser confocal scanning microscope. The procedure was the same as mentioned above for IRBP.

\section{Cell culture and treatments}

Y79 human retinoblastoma cells (ATCC HTB 18) were maintained in suspension in RPMI 1640 under standard conditions with $10 \%$ (vol./vol.) fetal bovine serum, penicillin $(100 \mathrm{U} / \mathrm{ml})$ and streptomycin $(100 \mu \mathrm{g} / \mathrm{ml})$ (Cultek, Barcelona, Spain) at $37^{\circ} \mathrm{C}$ and $5 \%$ (vol,/vol,) $\mathrm{CO}_{2}$. For glucose treatments, cells $\left(2 \times 10^{4} / \mathrm{ml}\right)$ were serum-starved and cultured for $72 \mathrm{~h}$ in increasing glucose concentrations $(5.5,10,15,25$ mmol D-glucose; Sigma) in RPMI 1640 with $0.1 \%$ (vol./vol.) fetal bovine serum. To rule out potential bias due to osmotic effect, D-mannitol (Sigma) was used at appropriate concentrations to equilibrate the osmosis. In another set of experiments, Y79 cells were plated on poly-D- 


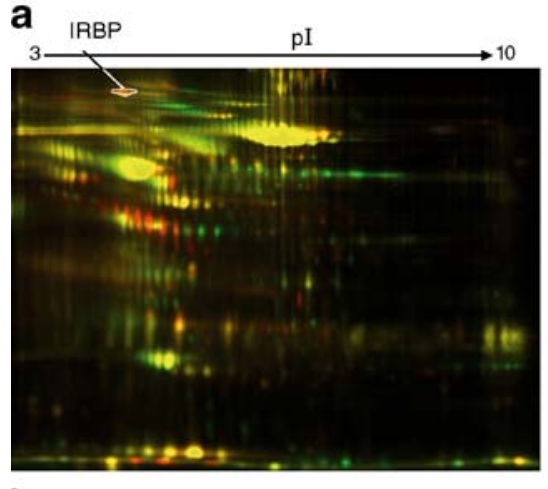

b

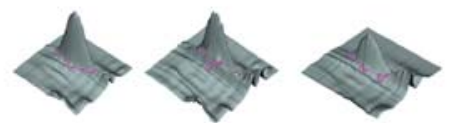

C

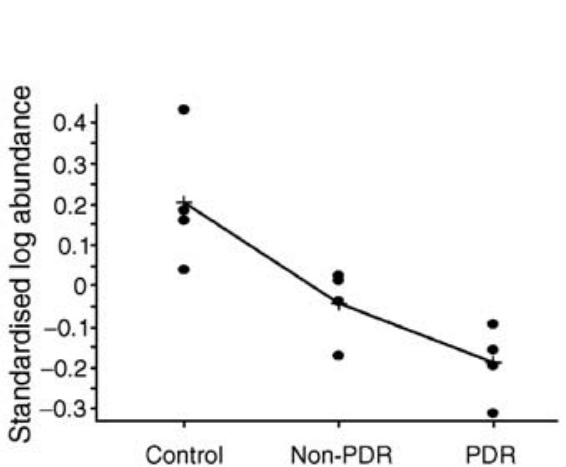

d

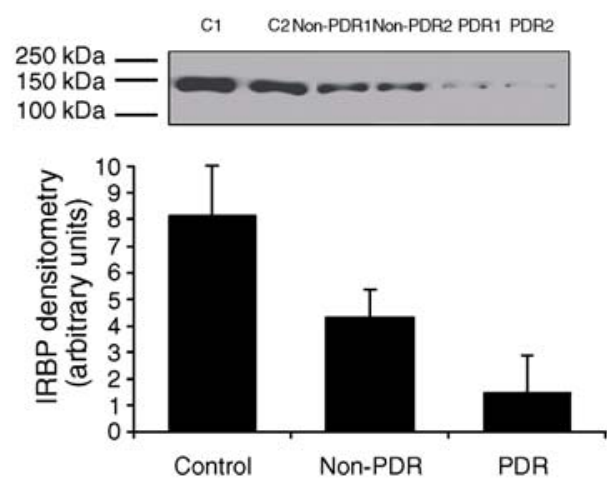

Fig. 1 a Superimposed images in pseudocolour from samples labelled with Cy3 (green, PDR vitreous proteins) and Cy5 (red, Control vitreous proteins), and run on a two-dimensional DIGE gel. The range of the horizontal dimension is in isoelectric points $(\mathrm{pI})$. The vertical dimension ranges from $15 \mathrm{kDa}$ (bottom) to $200 \mathrm{kDa}$ (top). The position of the spot corresponding to IRBP is marked. b Threedimensional images of the IRBP spot corresponding to the image of a Control, Non-PDR and PDR sample (left to right). c Standardised abundance plot for IRBP displaying the log of abundance observed for

lysine coated plates (BD, Madrid, Spain). After $24 \mathrm{~h}$ medium was replaced and either TNF- $\alpha(10,25$ and $50 \mathrm{ng} / \mathrm{ml})$ or IL$1 \beta$ (10 and $25 \mathrm{ng} / \mathrm{ml}$ ) (Prepro, London, UK) were added for additional $72 \mathrm{~h}$. To study IRBP expression, immunoblotting and real-time PCR were performed.

\section{Statistical analysis}

Results are expressed as mean $\pm \mathrm{SD}$. Comparisons between groups were performed using Student's $t$ test or ANOVA. Levels of statistical significance were set at $p<0.05$.

\section{Results}

IRBP in vitreous fluid

DIGE and western blot analyses The results for DIGE proteomic analysis of IRBP are summarised in Fig. 1a-c. To further validate these results, we assessed ten PDR, ten non-PDR and ten macular hole vitreous samples in addition to the samples used in DIGE. Intravitreous IRBP concentration (densitometric arbitrary units) was significantly lower in $\mathrm{PDR}<\mathrm{NPDR}<$ control $(1.95 \pm 1.63,4.47 \pm 2.30$, $8.19 \pm 3.41, p=0.03$ ) (Fig. 1d). As expected, IRBP was not detected in any serum sample (data not shown). This is because synthesis of IRBP is highly restricted to the neuroretina and pineal cells.

the spot in each of the four gel images corresponding to control, nonPDR and PDR samples, as indicated, after standardising the values using the internal standard pool images (Cy2) of each of the four gels. The line links the average abundance values for each group of samples (crosses). Student's $t$ test for the difference in abundance between each two groups results in $p<0.05$ in all cases. $\mathbf{d}$ Western blot analyses of vitreous samples corresponding to non-diabetic participants $(\mathrm{C} 1, \mathrm{C} 2)$, and non-PDR and PDR patients. Blots were quantified by densitometry, with bars representing the mean $\pm \mathrm{SD}$

\section{IRBP in retina}

$\beta$-Actin mRNA expression was similar between diabetic and non-diabetic retinas ( $p=\mathrm{NS}$ ). Thus, we calculated IRBP mRNA gene expression after normalising with $\beta$-actin.

$I R B P$ mRNA levels obtained in diabetic donors were significantly lower than those obtained from non-diabetic donors (1.16 \pm 0.93 vs $2.48 \pm 2.01, p=0.03$ ) (Fig. 2a).

Densitometric analysis of immunoblots showed lower IRBP levels in the retinas from diabetic donors than in

a

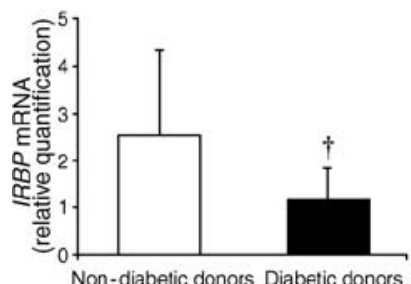

Fig. 2 a Real-time quantitative RT-PCR analysis of IRBP mRNA in human retinas from diabetic and non-diabetic donors. IRBP mRNA gene expression was calculated after normalising with $\beta$-actin. Bars represent the mean $\pm \mathrm{SD}, \dagger p=0.03$. b Western blot analyses of IRBP in human retina from non-diabetic and diabetic donors, with quantification by densitometry. Bars represent mean $\pm \mathrm{SD}, * * * p<0.001$ 

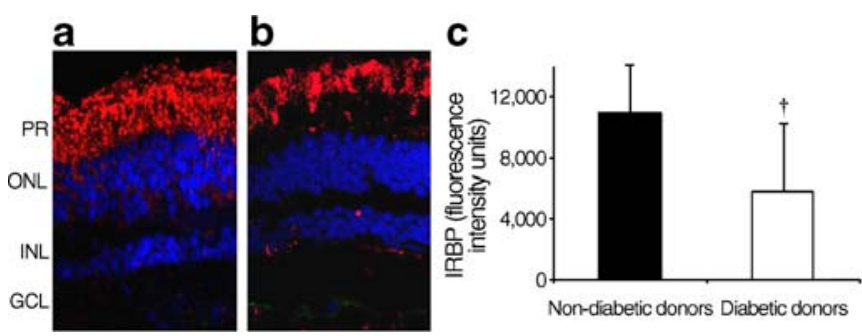

Fig. 3 Comparison of IRBP immunofluorescence (red) in representative samples from a non-diabetic donor (a) and a diabetic donor (b). GCL, ganglion cell layer; INL, inner nuclear layer; ONL, outer nuclear layer; PR, photoreceptors. Nuclei were stained using DAPI (blue). c Quantification of IRBP immunofluorescence in non-diabetic and diabetic retinas. Bars represent mean $\pm \mathrm{SD}, \dagger p=0.04$. Comparison of GFAP immunofluorescence (green) in representative samples from

those from non-diabetic donors $(2.58 \pm 0.69$ vs $6.14 \pm 1.17$, $p<0.001$ ) (Fig. 2b).

Laser scanning confocal images of IRBP immunofluorescence are shown in Fig. 3a-c. IRBP immunofluorescence was significantly lower in the retinas from diabetic donors than in those from non-diabetic donors $(5,787 \pm$ 4,531 vs $10,963 \pm 3,787, p=0.04$ ).

Effect of diabetes in retinal neurodegeneration: apoptosis and glial activation

To assess the presence of early diabetic damage in the retinas studied, we determined the rate of apoptosis and glial activation, two well-recognised markers of retinal neurodegeneration.

The percentage of apoptotic cells was higher in diabetic neuroretinas than in the age-matched non-diabetic retinas, but the differences, while having possible clinical consequences, were not statistically significant (outer nuclear layer $0.48 \pm 0.44 \%$ vs $0.05 \pm 0.06 \%, p=0.09$; inner nuclear layer $2.23 \pm 2.04$ vs $1.29 \pm 2.31, p=0.34$; ganglionar cell layer $2.97 \pm 0.60 \%$ vs $1.30 \pm 0.43 \%, p=0.08$ ). Neuroglial activation was demonstrated in diabetic eyes by an increase of GFAP production in the neuroretina. GFAP immunofluorescence was significantly higher in diabetic than in non-diabetic retinas $(3,123 \pm 418$ vs $1,119 \pm 489, p<0.01)$ (Fig. 3d-f).

Effect of high glucose and cytokines on IRBP production in vitro

Glucose caused a dose-dependent reduction of IRBP mRNA expression and IRBP content in human Y79 retinoblastoma cultures (Fig. 4a, b). TNF- $\alpha$ and IL-1 $\beta$ also decreased IRBP production by human Y79 retinoblastoma cells (Fig. 5a-d).
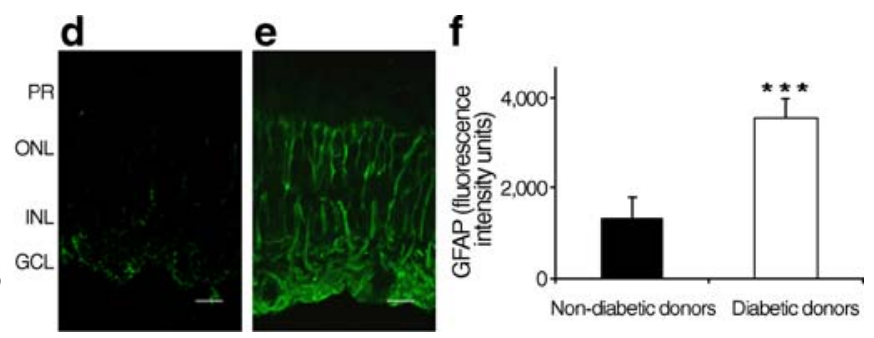

a non-diabetic donor (d) and a diabetic donor (e). Key, as above (a, b). In the diabetic retina, the Müller cells' endfeet show abundant GFAP immunofluorescence and the radial processes stain intensely throughout the inner and outer retina. Scale bar, $20 \mu \mathrm{m}$. f Quantification of GFAP immunofluorescence in non-diabetic and diabetic retinas. Bars represent mean $\pm \mathrm{SD},{ }^{* * *} p<0.001$

\section{Discussion}

IRBP, a large glycoprotein highly restricted to the interphotoreceptor matrix, facilitates the transport of retinoids between the photoreceptor outer segments and RPE and therefore plays a major role in the visual cycle [7, 8]. In addition it is involved in the maintenance of photoreceptors $[9,10]$. In the

a

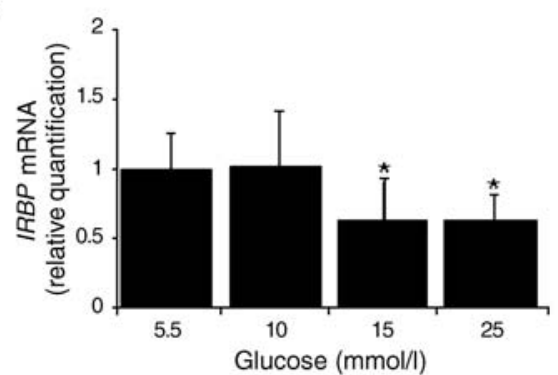

b

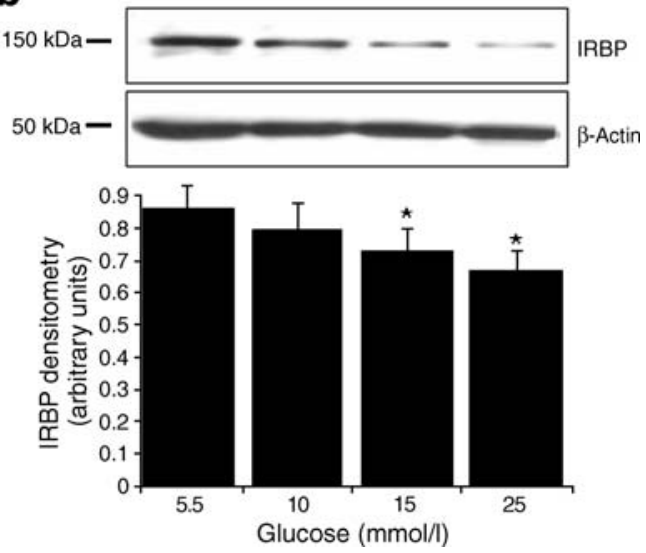

Fig. 4 Effect of different glucose concentrations (5.5, 10, 15 and $25 \mathrm{mmol}$ ) on IRBP mRNA levels (RT-PCR) (a) and protein content (b) in Y79 cell cultures, which is shown as blot and quantified by densitometry. Bars represent mean $\pm \mathrm{SD},{ }^{*} p<0.05$ vs control $(5.5 \mathrm{mmol})$ 
Fig. 5 Effect of treatment with TNF- $\alpha(10,25$ and $50 \mathrm{ng} / \mathrm{ml})$ and IL- $1 \beta$ (10 and $25 \mathrm{ng} / \mathrm{ml})$ on IRBP mRNA levels (RT-PCR) $(\mathbf{a}, \mathbf{b})$ and protein content (western blot and quantification by densitometry) (c, d) in Y79 cell cultures. FBS, fetal bovine serum. Control: RPMI 1640 1\% FBS. Bars represent mean $\pm \mathrm{SD}$, ${ }^{*} p<0.05$ vs control a

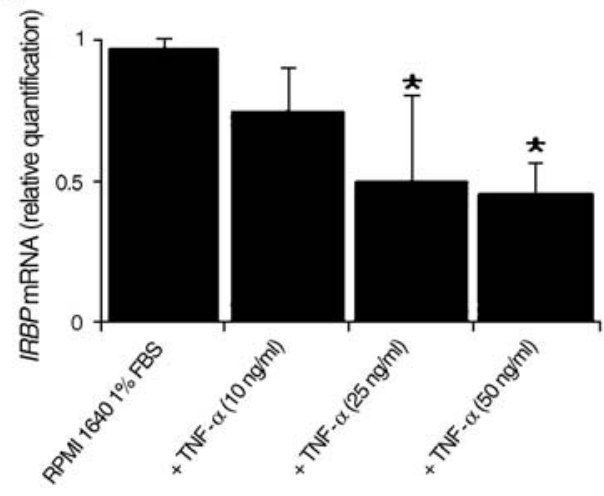

C
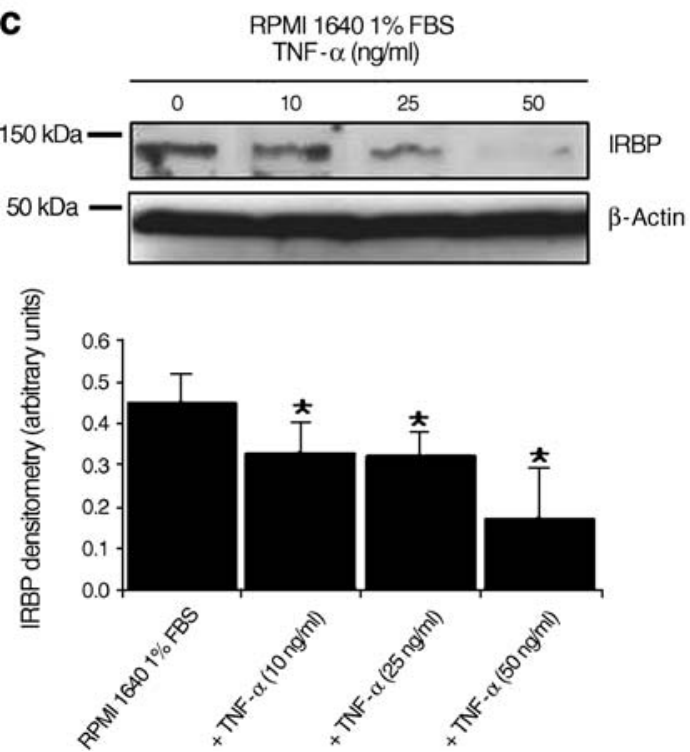

b

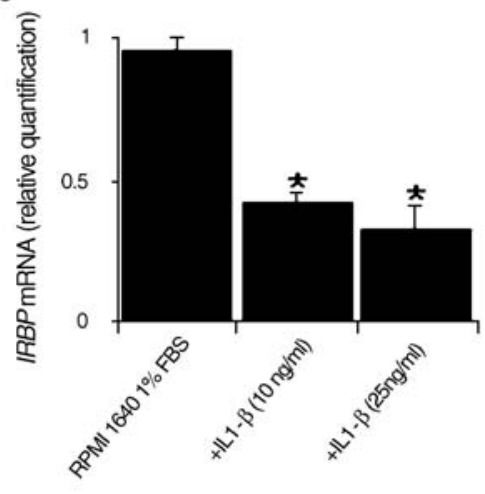

d
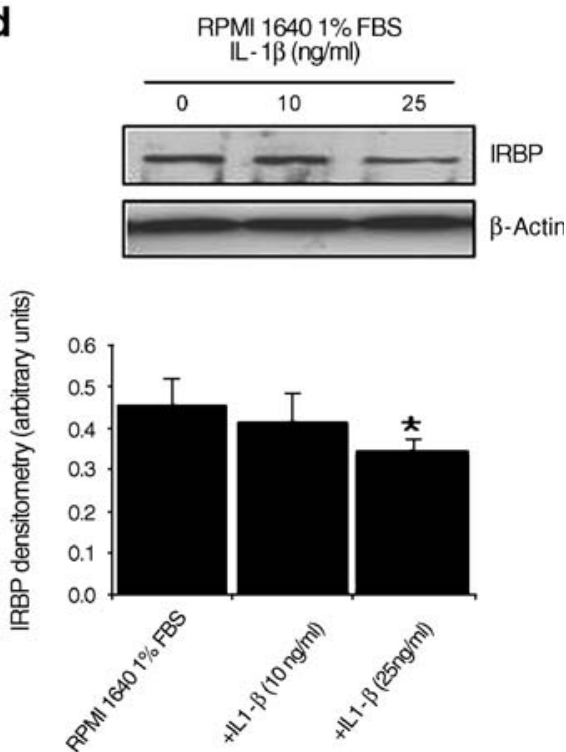

present study we provide evidence that downregulation of $I R B P$ is an early event in the human diabetic retina.

One of the limiting factors in diabetic retinopathy research is the impossibility, for obvious reasons, of obtaining retinal biopsies from diabetic patients. Since retinal biopsy is not available, vitreous fluid from diabetic patients undergoing vitreoretinal surgery is currently used to indirectly explore the events taking place in the retina. However, because most vitrectomies are indicated in PDR, the results obtained in the study of vitreous fluid are only valid for advanced stages of diabetic retinopathy. In the present study, we obtained vitreous fluid from diabetic patients with mild or moderate NPDR, who underwent vitreoretinal surgery for other reasons unrelated to diabetic retinopathy. This approach enabled us to demonstrate for the first time that patients with NPDR have lower intravitreous IRBP levels than non-diabetic patients. Because disruption of the blood-retinal barrier is an early event in the pathogenesis of diabetic retinopathy, one of the confounding factors when interpreting results obtained in vitreous fluid measurements is that they can also reflect serum levels, rather than local production by the retina. However, given that IRBP is only produced by photoreceptors and pineal cells [7, 19], intravitreous IRBP concentration is a reliable index of its retinal synthesis.

In addition, we found that expression and content of IRBP were lower in retinas from diabetic donors than in those of non-diabetic donors. Therefore, there is a low abundance of IRBP in the diabetic eye. Given that our diabetic donors were free of ophthalmoscopic abnormalities, it could be argued that retinas from these patients were without any diabetes-related damage. However, this is not the case, because glial activation, a characteristic feature of diabetic retinal neurodegeneration, was present. Furthermore, the higher, but not significant rate of apoptosis observed in the neuroretinas from diabetic donors strongly suggests that an IRBP deficit exists in very early stages of diabetic retinopathy.

The mechanisms leading to reduced amounts of IRBP in the diabetic eye could not be determined with the current data. IRBP is a dominant antigen in autoimmune uveitis [20]. Recently, moreover, proteolysis of IRBP has been shown to occur [21]. The loss of IRBP detected in the 
vitreous fluid of diabetic patients could be a consequence of increased proteolytic activity due to leucocyte-released proteases $[22,23]$. In fact, due to the anatomical bloodretinal barriers, IRBP has a hidden nature, but the disruption of the blood-retinal barrier that occurs in diabetic retinopathy facilitates antigen presentation and the subsequent activation of autoreactive T cells [21]. Therefore, it is possible that IRBP acts as a first trigger of the inflammatory process present in the early stages of diabetic retinopathy and that the lower levels of IRBP detected in the diabetic eye are the consequence of increased proteolytic activity secondary to inflammation. However, we found low mRNA levels of $I R B P$ in the retinas of diabetic donors and, in consequence, lower production, rather than increased catabolism seems to be the primary cause of the IRBP deficit detected in the diabetic eye. In addition, high glucose levels and proinflammatory cytokines were able to downregulate IRBP expression and IRBP content in human Y79 retinoblastoma cells, suggesting that the diabetic milieu might play an essential role in the underproduction of IRBP in diabetic patients. Nevertheless, it should be noted that lower expression of IRBP was found in Y79 cells in comparison with non-transformed cells. Therefore, our results cannot be readily extrapolated to the clinical setting. As a result, further studies in primary cultures of photoreceptors are needed.

Neurodegeneration is an early event in the pathogenesis of diabetic retinopathy, antedating and participating in the microcirculatory abnormalities that occur in the condition $[2$, $3,24]$. Neuroretinal damage produces functional abnormalities such as colour vision defects, reduced contrast sensitivity and abnormalities in dark adaptation. These alterations can occur before microvascular lesions are detectable in ophthalmological examinations, but they tend to be predictive of PDR, which ultimately leads to degenerative changes and significant visual impairment [24-26]. The design of our study does not allow us to say whether the lower IRBP content in diabetic retinas is a cause or a consequence of retinal neurodegeneration. However, given that IRBP has neuroprotective properties, it is possible that lower production of IRBP participates in diabetic retinal neurodegeneration. In addition, since IRBP is synthesised by photoreceptors, the higher apoptosis rate detected in the photoreceptor layer could be involved in lower IRBP production, thus initiating a vicious circle that could be added to the classical pathways involved in diabetic retinopathy development.

In conclusion, lower IRBP production is an early event in the human diabetic retina and is associated with retinal neurodegeneration. Since IRBP plays a major role in the visual cycle and is essential for photoreceptor survival, an IRBP deficit could be involved in the pathogenesis of diabetic retinopathy. However, further studies are required to determine the cause of IRBP underproduction in the diabetic retina and its clinical implications.
Acknowledgements This study was supported by grants from Novo Nordisk Pharma, the Ministerio de Ciencia e Innovación (MCIN) (SAF2006-05284 and SAF2009-07408), the Fundación para la Diabetes and the Generalitat de Catalunya (2005SGR-00030), and CIBERDEM, which is an initiative of the Instituto de Salud Carlos III. M. Villarroel is recipient of a grant from the Fundació Institut de Recerca Hospital Universitari Vall d'Hebron. We are grateful to J. M. Nickerson, Emory University, USA, for providing us with the antibodies against IRBP.

Duality of interest The authors declare that there is no duality of interest associated with this manuscript.

\section{References}

1. Barber AJ, Lieth E, Khin SA, Antonetti DA, Buchanan AG, Gardner TW (1998) Neural apoptosis in the retina during experimental and human diabetes. Early onset and effect of insulin. J Clin Invest 102:783-791

2. Lieth E, Gardner TW, Barber AJ, Antonetti DA, Penn State Retina Research Group (2000) Retinal neurodegeneration: early pathology in diabetes. Clin Experiment Ophthalmol 28:3-8

3. Lorenzi M, Gerhardinger C (2001) Early cellular and molecular changes induced by diabetes in the retina. Diabetologia 44:791-804

4. Antonetti DA, Barber AJ, Bronson SK, JDRF Diabetic Retinopathy Center Group et al (2006) Diabetic retinopathy: seeing beyond glucose-induced microvascular disease. Diabetes 55:2401-2411

5. Carrasco E, Hernández C, Miralles A, Huguet P, Farrés J, Simó R (2007) Lower somatostatin expression is an early event in diabetic retinopathy and is associated with retinal neurodegeneration. Diabetes Care 30:2902-2908

6. Carrasco E, Hernández C, de Torres I, Farrés J, Simó R (2008) Lowered cortistatin expression is an early event in the human diabetic retina and is associated with apoptosis and glial activation. Mol Vis 14:1496-1502

7. Gonzalez-Fernandez F (2003) Interphotoreceptor retinoid-binding protein - an old gene for new eyes. Vis Res 43:3021-3036

8. Wu Q, Blakeley LR, Cornwall MC, Crouch RK, Wiggert BN, Koutalos Y (2007) Interphotoreceptor retinoid-binding protein is the physiologically relevant carrier that removes retinol from rod photoreceptor outer segments. Biochemistry 24:8669-8679

9. Gonzalez-Fernandez F, Ghosh D (2008) Focus on molecules: interphotoreceptor retinoid-binding protein (IRBP). Exp Eye Res 86:169-170

10. Liou GI, Fei Y, Peachey NS, Matragoon S et al (1998) Early onset photoreceptor abnormalities induced by targeted disruption of the interphotoreceptor retinoid-binding protein gene. J Neurosci $18: 4511-4520$

11. Narfström K, Nilsson SE, Wiggert B, Lee CGJ, van Veen $T$ (1989) Reduced level of interphotoreceptor retinoid-binding protein (IRBP), a possible cause for retinal degeneration in the Abyssinian cat. Cell Tissue Res 257:631-639

12. Wiggert B, van Veen T, Kutty G et al (1994) An early decrease in IRBP gene expression in Abyssinian cats homozygous for hereditary rod-cone degeneration. Cell Tissue Res 278:291-298

13. Ripps H, Peachey NS, Xu X, Nozell SE, Smith SB, Liou GI (2000) The rhodopsin cycle is preserved in IRBP "knockout" mice despite abnormalities in retinal structure and function. Vis Neurosci 17:97-105

14. den Hollander A, McGee TL, Zivello C et al (2009) A homozygous missense mutation in the IRBP gene (RBP3) associated with autosomal recessive retinitis pigmentosa. Invest Ophthalmol Vis Sci 50:1864-1872 
15. Garcia-Ramírez M, Canals F, Hernández C et al (2007) Proteomic analysis of human vitreous fluid by fluorescence-based difference gel electrophoresis (DIGE): a new strategy for identifying potential candidates in the pathogenesis of proliferative diabetic retinopathy. Diabetologia 50:1294-1303

16. Wilkinson CP, Ferris FL 3rd, Klein RE et al (2003) Proposed international clinical diabetic retinopathy and diabetic macular edema disease severity scales. Ophthalmology 110:1677-1682

17. American Diabetes Association (2004) Nephropathy in diabetes. Diabetes Care 27:S79-S83

18. Hernández C, Carrasco E, Casamitjana R, Deulofeu R, GarcíaArumí J, Simó R (2005) Somatostatin molecular variants in the vitreous fluid: a comparative study between diabetic patients with proliferative diabetic retinopathy and nondiabetic control subjects. Diabetes Care 28:1941-1947

19. Lopes MB, Gonzalez-Fernandez F, Scheithauer BW, VandenBerg SR (1993) Differential expression of retinal proteins in a pineal parenchymal tumor. J Neuropathol Exp Neurol 52:516-524

20. Wang M, Bai F, Pries M, Buus S, Prause JU, Nissen MH (2006) Identification of MHC class I H-2 Kb/Db-restricted immunogenic peptides derived from retinal proteins. Invest Ophthalmol Vis Sci 47:3939-3945

21. Descamps FJ, Kangave D, Cauwe B et al (2008) Interphotoreceptor retinoid-binding protein as biomarker in systemic autoimmunity with eye inflictions. J Cell Mol Med 12:2449-2456

22. Giebel SJ, Menicucci G, McGuire PG, Das A (2005) Matrix metalloproteinases in early diabetic retinopathy and their role in alteration of the blood-retinal barrier. Lab Invest 85:597-607

23. Jin M, Kashiwagi K, Iizuka Y, Tanaka Y, Imai M, Tsukahara S (2001) Matrix metalloproteinases in human diabetic and nondiabetic vitreous. Retina 2:28-33

24. Barber AJ (2003) A new view of diabetic retinopathy: a neurodegenerative disease of the eye. Prog Neuropsychopharmacol Biol Psychiatry 27:283-290

25. Ewing FM, Deary IJ, Strachan MW, Frier BM (1998) Seeing beyond retinopathy in diabetes: electrophysiological and psychophysical abnormalities and alterations in vision. Endocr Rev 19:462-476

26. Shirao Y, Kawasaki K (1998) Electrical responses from diabetic retina. Prog Retin Eye Res 17:59-76 